\title{
Derivation of Loop Gain and Stability Test for Multiple Feedback Low-pass Filter Using Deboo Integrator
}

\author{
MinhTri Tran*, Anna Kuwana, and Haruo Kobayashi \\ Division of Electronics and Informatics, Gunma University, Kiryu 376-8515, Japan \\ *Email: trantri.ks@gmail.com
}

\begin{abstract}
Proposed derivation and measurement of self-loop function for a second-order multiple feedback low-pass filter using Deboo integrator are introduced. The self-loop function of the filter is derived and analyzed based on the widened superposition principle. An alternating current conservation measurement is proposed to measure the self-loop function. Research results show that the selected passive components (resistors, capacitors) of the frequency compensation of Miller capacitors in the operational amplifier and in the multiple feedback low-pass filter can cause a damped oscillation noise when the stable conditions for the transfer functions of these networks are not satisfied.
\end{abstract}

Keywords: Widened Superposition, Stability Test, Self-loop Function, Multiple Feedback LPF, Voltage Injection.

\section{Introduction}

Analog active filters are widely applied in modern electronics $^{(1)}$. One of the most famous active filters is the multiple feedback low-pass filter (MFB LPF) ${ }^{(2)}$. Although the circuit was introduced since many years it is still receiving interest of researchers in modifying the frequency characteristics of this circuit to fit the new CMOS technology ${ }^{(3)}$. Based on current trends in analog signal processing system development, the most important objective in transient stability process is to ensure global stability of large interconnected filters ${ }^{(4)}$. Moreover, feedback control theories are widely applied in the processing of analog signals ${ }^{(5)}$. In conventional analysis of a feedback system, the term of "A $\beta(\mathrm{s})$ " is called loop gain when the denominator of the transfer function is simplified as $1+\mathrm{A} \beta(\mathrm{s})$, where $\mathrm{A}(\mathrm{s}), \beta(\mathrm{s})$, are the open loop gain, and feedback gain, respectively. The stability of a feedback network is determined by the magnitude and phase plots of the loop gain. However, a passive filter is not a closed loop system. Furthermore, the denominator of the transfer function of an analog filter, regardless of active or passive is also simplified as $1+\mathrm{L}(\mathrm{s})$, where $\mathrm{L}(\mathrm{s})$ is called "self-loop function". Therefore, the term of "self-loop function" is proposed to define $\mathrm{L}(\mathrm{s})$ for both cases with and without feedback filters. This paper provides an introduction to the derivation of the transfer function, the measurement of self-loop function and stability test for a second-order multiple feedback low-pass filter based on the alternating current conservation measurement and the widened superposition principle. In the research not only the results of the mathematical models but also the results of simulation of the designed circuits are provided, including the stability test.

The main contribution of this paper comes from the stability test for a second-order multi feedback low-pass filter using the Deboo integrator based on the widened superposition and the voltage injection technique. The background knowledge is presented in Section 2. Section3 mathematically analyzes illustrative first-and second-order denominator complex functions considered in details. Section4 presents the stability test for a mathematical model of the two-stage operational amplifier which is used in the multiple feedback circuit. SPICE simulation results and the stability test for the proposed design filter are described in Section 5. A brief discussion of the research results is given in Section 6. The main points of this work are summarized in Section 7. We have collected a few important notions and results from analysis in Appendix for easy references.

\section{Design considerations for multiple feedback low-pass filter}




\subsection{Widened superposition principle}

In this section, we propose a new concept of the superposition principle which is useful when we derive the transfer function of a network. The conventional superposition theorem is used to find the solution to linear networks consisting of two or more sources (independent sources, linear dependent sources) that are not in series or parallel. To consider the effects of each source independently requires that sources be removed and replaced without affecting the final result. To remove a voltage source when applying this theorem, the difference in potential between the terminals of the voltage source must be set to zero (short circuit); removing a current source requires that its terminals be opened (open circuit). This procedure is followed for each source in turn, and then the resultant responses are added to determine the true operation of the circuit. There are some limitations of conventional superposition theorem. Superposition cannot be applied to power effects because the power is related to the square of the voltage across a resistor or the current through a resistor. Superposition theorem cannot be applied for non-linear circuit (diodes or transistors). In order to calculate the load current or the load voltage for the several choices of the load resistance of the resistive network, one needs to solve for every source voltage and current, perhaps several times. With the simple circuit, this is fairly easy but in a large circuit this method becomes a painful experience.

In the paper, the nodal analysis on circuits is used to obtain multiple Kirchhoff current law equations. The term of "widened superposition" is proposed to define a general superposition principle which is the standard nodal analysis equation, and simplified for the case when the impedance from node A to ground is infinity and the current injection into node $\mathrm{A}$ is 0 . In a circuit having more than one independent source, we can consider the effects of all the sources at a time. The widened superposition principle is used to derive the transfer function of a network ${ }^{(6,7)}$. Energy at one place is proportional with their input sources and the resistance distances of transmission spaces. Let $E_{A}(t)$ be energy at one place of multi-sources $E_{i}(t)$ which are transmitted on the different resistance distances $d_{i}\left(\mathrm{R}, \mathrm{Z}_{\mathrm{L}}\right.$, and $Z_{C}$ in electronic circuits) of the transmission spaces as shown in Fig. 1. Widened superposition principle can be defined as

$$
E_{A}(t) \sum_{i=1}^{n} \frac{1}{d_{i}}=\sum_{i=1}^{n} \frac{E_{i}(t)}{d_{i}}
$$

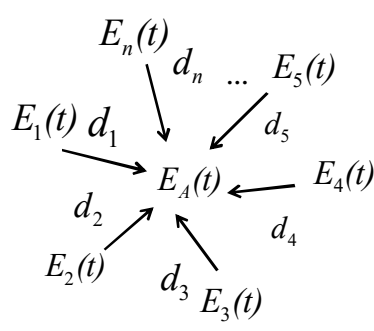

Fig. 1. Energy at one node based on superposition principle.

The import of these concepts into circuit theory is relatively new with much recent progress regarding filter theory, analysis and implementation.

\subsection{Complex function}

In this section, we describe a transfer function as the form of a complex function where the variable is an angular frequency. In frequency domain, the transfer function and the self-loop function of a filter are complex functions. Complex functions are typically represented in two forms: polar or rectangular.The polar form and the rectangular representation of a complex function $\mathrm{H}(\mathrm{j} \omega)$ is written as

$$
\begin{aligned}
H(j \omega) & =\operatorname{Re}\{H(j \omega)\}+j \operatorname{Im}\{H(j \omega)\} \\
& =\sqrt{(\operatorname{Re}\{H(j \omega)\})^{2}+(\operatorname{Im}\{H(j \omega)\})^{2}} e^{j \arctan \left(\frac{\operatorname{Im}\{H(j \omega)\}}{\operatorname{Re}\{H(j \omega)\}}\right)}
\end{aligned}
$$

where $\operatorname{Re}\{\mathrm{H}(\mathrm{j} \omega)\}$ is the real part of $\mathrm{H}(\mathrm{j} \omega)$ and $\operatorname{Im}\{\mathrm{H}(\mathrm{j} \omega)\}$ is the imaginary part of $\mathrm{H}(\mathrm{j} \omega)$, and $\mathrm{j}$ is the imaginary operator $\mathrm{j}^{2}=-1$. The real quantity $\sqrt{(\operatorname{Re}\{H(j \omega)\})^{2}+(\operatorname{Im}\{H(j \omega)\})^{2}}$ is known as the amplitude or magnitude, the real quantity $\left.\arctan \left(\frac{\operatorname{Im}\{H(j \omega)\}}{\operatorname{Re}\{H(j \omega)\}}\right\}\right)$ is called the angle $\angle H(j \omega)$, which is the angle between the real axis and $H(j \omega)$. The angle may be expressed in either radians or degrees and real quantity $\frac{\operatorname{Im}\{H(j \omega)\}}{\operatorname{Re}\{H(j \omega)\}}$ is called the argument $\operatorname{Arg}\{H(j \omega)\}$ which is the ratio between the real part and the imaginary part of $\mathrm{H}(\mathrm{j} \omega)$. The operations of addition, subtraction, multiplication, and division are applied to complex functions in the same manner as that they are to complex numbers.

Complex functions are typically expressed in three forms: magnitude-angular plots (Bode plots), polar charts (Nyquist charts), and magnitude-argument diagrams (Nichols diagrams). In the paper, the stability test is performed on the magnitude-angular charts.

\subsection{Graph signal model for complex function}


In this section, we describe the graph signal model of a typical complex function which is the same as the graph signal model of a feedback system. A negative-feedback amplifier is an electronic amplifier that subtracts a fraction of its output from its input, so that negative feedback opposes the original signal. The applied negative feedback can improve its performance (gain stability, linearity, frequency response, step response) and reduce sensitivity to parameter variations due to manufacturing or environment. Thanks to these advantages, many amplifiers and control systems use negative feedback. However, the denominator complex functions are also expressed in the graph signal model which is the same as the negative feedback system. A general denominator complex function is rewritten as

$$
H(s)=\frac{V_{\text {out }}(s)}{V_{\text {in }}(s)}=\frac{A(s)}{1+L(s)}
$$

This form is called the standard form of the denominator complex function. The output signal is calculated as

$$
V_{\text {out }}(s)=A(s)\left[V_{\text {in }}(s)-\frac{L(s)}{A(s)} V_{\text {out }}(s)\right]
$$

Fig. 2 presents the graph signal model of a general denominator complex function. The feedback system is unstable if the closed-loop "gain" goes to infinity, and the circuit can amplify its own oscillation. The condition for oscillation is

$$
L(s)=-1=1 e^{-j \pi(2 k+1)} ; k \in Z
$$

Through the self-loop function, a second-order denominator complex function can be found that is stable or not. The concepts of phase margin and gain margin are used to asset the characteristics of the loop function at unity gain. The conventional test of the loop gain, which is called "Barkhausen's criteria", is unity gain and $-180^{\circ}$ of phase in magnitude-phase plots (Bode plots) ${ }^{(8)}$.

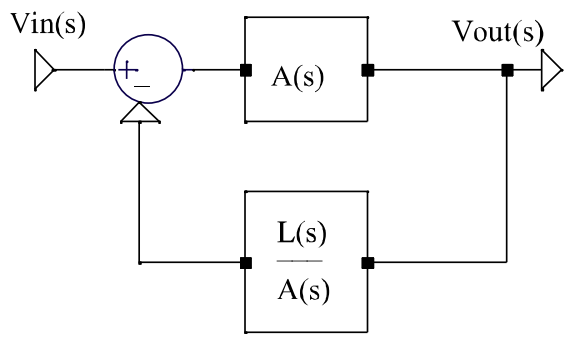

Fig.2. Graph signal model of general complex function.

\subsection{Alternating current conservation measurement}

In this section, we describe a mathematical way to measure the self-loop function based on the alternating current conservation when we inject an alternating signal sources (alternating current or voltage sources) and connect the input of the network into the alternating current ground (AC ground). In general, the term of "alternating current conservation" is proposed to define this technique. The main idea of this method is that the alternating current is conserved. In other words, at the output node the incident alternating current is equal to the transmitted alternating current. If we inject a alternating current source (or alternating voltage source) at the output node, the self-loop function can be derived by ratio of the incident voltage $\left(\mathrm{V}_{\text {inc }}\right.$ ) and the transmitted voltage $\left(\mathrm{V}_{\text {trans }}\right)$ as shown in Fig. 3. Compared to measurement results of the alternating current conservation with the conventional ones (voltage injection), they are the same.

In order to break the feedback loop without disturbing the signal termination conditions, and ensure that the loop is opened for AC signals, a balun transformer inductor can be used to isolate the signal source from the original network as shown in Fig. 4. In this case, the values of resistors and inductors are very large. Compared to the proposed measurement with the conventional replica measurement, they are the same measurement results. Apply the widened superposition principle at $\mathrm{V}_{\text {inc }}$ and $\mathrm{V}_{\text {trans }}$ nodes, and the self-loop function is derived as

$$
\frac{V_{\text {inc }}}{A(s)}=\frac{L(s)}{A(s)} V_{\text {trans }} \Rightarrow L(s)=\frac{V_{\text {inc }}}{V_{\text {trans }}}
$$

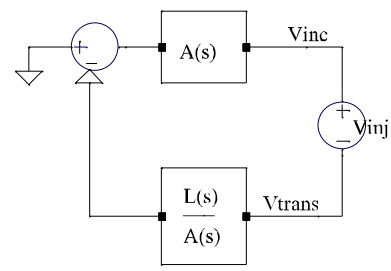

(a)

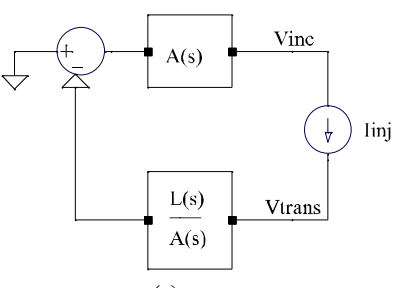

(c)
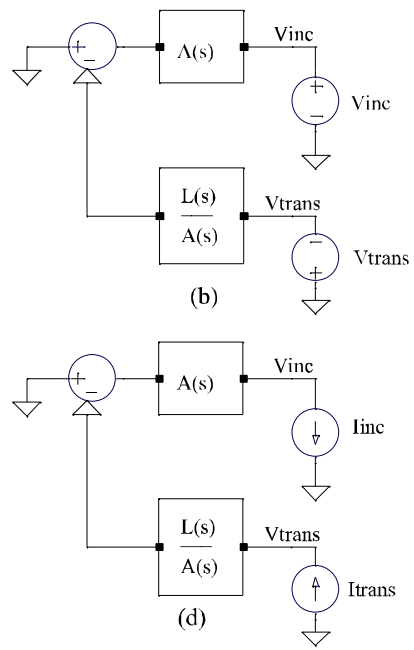

Fig. 3. Derivation of self-loop function based on alternating current conservation. 


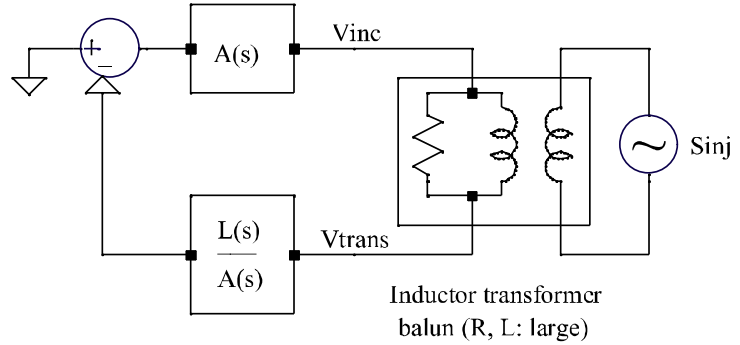

Fig. 4. Derivation of self-loop function based on balun transformer inductor injection method.

\section{Analysis of first- and second-order denominator complex functions}

\subsection{First-order denominator complex function}

In this section, we shall present the frequency response of a typical first-order denominator complex function on the magnitude-angular charts. A general transfer function of the first-order denominator complex function is defined as in Eq. (7). Assume that the constant variable $a$ is not equal to zero. If the constant is smaller than zero, it is expressed as a complex number $\left(a<0 \Rightarrow a=|a| j^{2}=|a| e^{ \pm j \pi}\right)$. In this paper, the angular of the constant is not written in details.

$$
H(s)=\frac{1}{a s+b}
$$

If the constant $b$ is equal to zero, the complex function is

$$
H(s=j \omega)=\frac{1}{j a \omega}=\frac{1}{a \omega} e^{j \frac{\pi}{2}}
$$

This complex function is stable, because the angular is a constant (90 degrees). When the constant $b$ is not equal to zero, the simplified form of Eq. (7) is

$$
H(s=j \omega)=\frac{1}{b} \frac{1}{\left(1+\frac{a}{b} s\right)}=\frac{1}{b} \frac{1}{\sqrt{1+\left(\frac{a}{b} \omega\right)^{2}}} e^{j \arctan \left(-\frac{a}{b} \omega\right)}
$$

Here, the cut-off angular frequency is $\omega_{c u t}=\frac{b}{a}$. The magnitude of the complex function decreases very fast from the cut-off angular frequency. Therefore, the high-order harmonics of a step input are significantly reduced when it goes into the network.

\subsection{Self-loop function of first-order denominator complex function}

In this section, we investigate the characteristics of the self-loop function $\mathrm{L}(\mathrm{s})$ of the first-order denominator complex function on the magnitude-angular charts. From Eq. (8), the self-loop function is

$$
L(j \omega)=j \frac{a}{b} \omega=\frac{a}{b} \omega e^{j \frac{\pi}{2}}
$$

Through the self-loop function, it is easy to find that the first-order denominator complex function is stable, because the phase does not change when the angular frequency goes from 0 to infinity.

\subsection{Second-order denominator complex functions}

In this section, we shall analyze the frequency response of a typical second-order denominator complex function on the magnitude-angular charts. A general second-order denominator complex function is defined as in Eq. (11). Assume that all constant variables are not equal to zero.

$$
H(s=j \omega)=\frac{1}{a s^{2}+b s+c}
$$

From Eq. (26) in Appendix A.1, the simplified complex function is

$$
H(j \omega)=\frac{\frac{4 a}{b^{2}}}{\left(1+j \frac{2 a}{b} \omega\right)^{2}+\left(\frac{2 a}{b}\right)^{2}\left[\frac{c}{a}-\left(\frac{b}{2 a}\right)^{2}\right]}
$$

In order to plot the magnitude-angular charts, the values of magnitude-angular of the complex function, which are calculated in Appendix A.1, are summarized on Table 1. In overdamped case, the magnitude of the complex function is so high from the first cut-off angular frequency $\omega_{\text {cut } 1}=\left|\frac{b}{2 a}\left(1-\frac{2 a}{b} \sqrt{\frac{c}{a}-\left(\frac{b}{2 a}\right)^{2}}\right)\right|$ to the second cut-off angular frequency $\omega_{\text {cut } 2}=\left|\frac{b}{2 a}\left(1-\frac{2 a}{b} \sqrt{\frac{c}{a}-\left(\frac{b}{2 a}\right)^{2}}\right)\right|$. Therefore, this gain will amplify the high order harmonics from $\omega_{\text {cut1 }}$ to $\omega_{\text {cut2 }}$ of an input signal which includes many harmonics.

\subsection{Damped oscillation noise}

In this section, we describe the response of a typical second-order denominator complex function to a step input or a square wave. Based on the Fourier series expansion of the square wave, the waveforms of the pulse wave are expressed in many functions of time with many different 
frequencies as shown in Fig. 5. The waveform function of a square wave is

$$
S(t)=\frac{4}{\pi} \sum_{k=1}^{\infty} \frac{\sin \left(2 \pi(2 k-1)\left(f_{1}\right) t\right)}{2 k-1}
$$

- In under-damped case, the high-order harmonics of the step signal are significantly reduced from the first cut-off angular frequency. Therefore, the rising time and falling timeare rather short. In this case, the system is absolutely stable.

- In case of critically damped, the rising time and falling time are longer than the underdamped case. Now, the system is marginally stable. The energy propagation is also maximal because this condition is equal to the balanced charge-discharge time condition ${ }^{(9)}$.

- In over-damped case of the complex function, the gain at the cut-off angular frequency will amplify the high-order harmonics of the step signal that causes the peaking or ringing. Ringing is an unwanted oscillation of a voltage or current. The term of "damped oscillation noise" is proposed to define this unwanted oscillation which fades away with time, particularly in the step response (the response to a sudden change in input). Damped oscillation noise is undesirable because it causes extra current to flow, which leads to thereby wasting energy and causing extra heating of the components. It can cause unwanted electromagnetic radiation to be emitted. Therefore, the system is unstable.
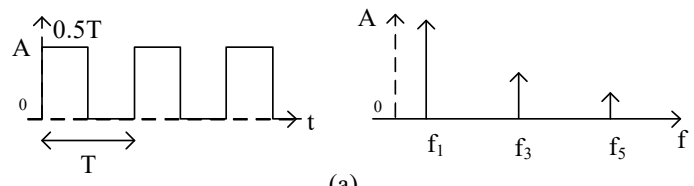

(a)
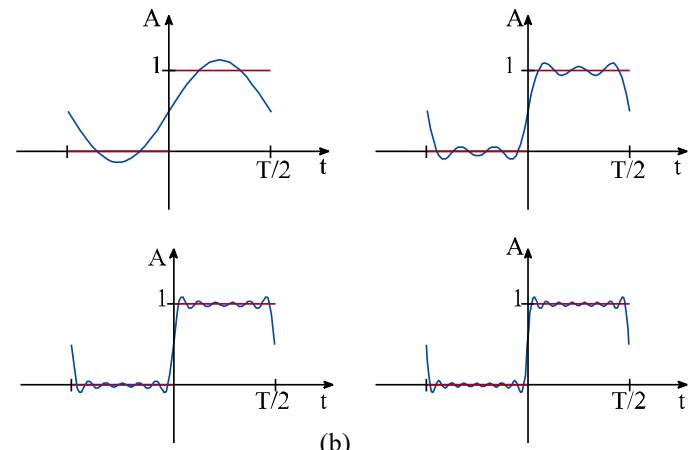

(b)

Fig. 5. Square wave: (a) waveform, spectrum, and (b) partial sums of Fourier series.

\subsection{Self-loop function of second-order denominator complex function}

In this section, we investigate the characteristics of the self-loop function L(s) on the magnitude-angular charts. The general transfer function and self-loop function are rewritten as in Eq. (14). The values of magnitude and angular of the self-loop function, which are calculated in Appendix A.2, are summarized in Table 2. In this work, the self-loop function is only sketched on the magnitude-angular charts.

Table 1. Summary of magnitude-angular values of transfer function

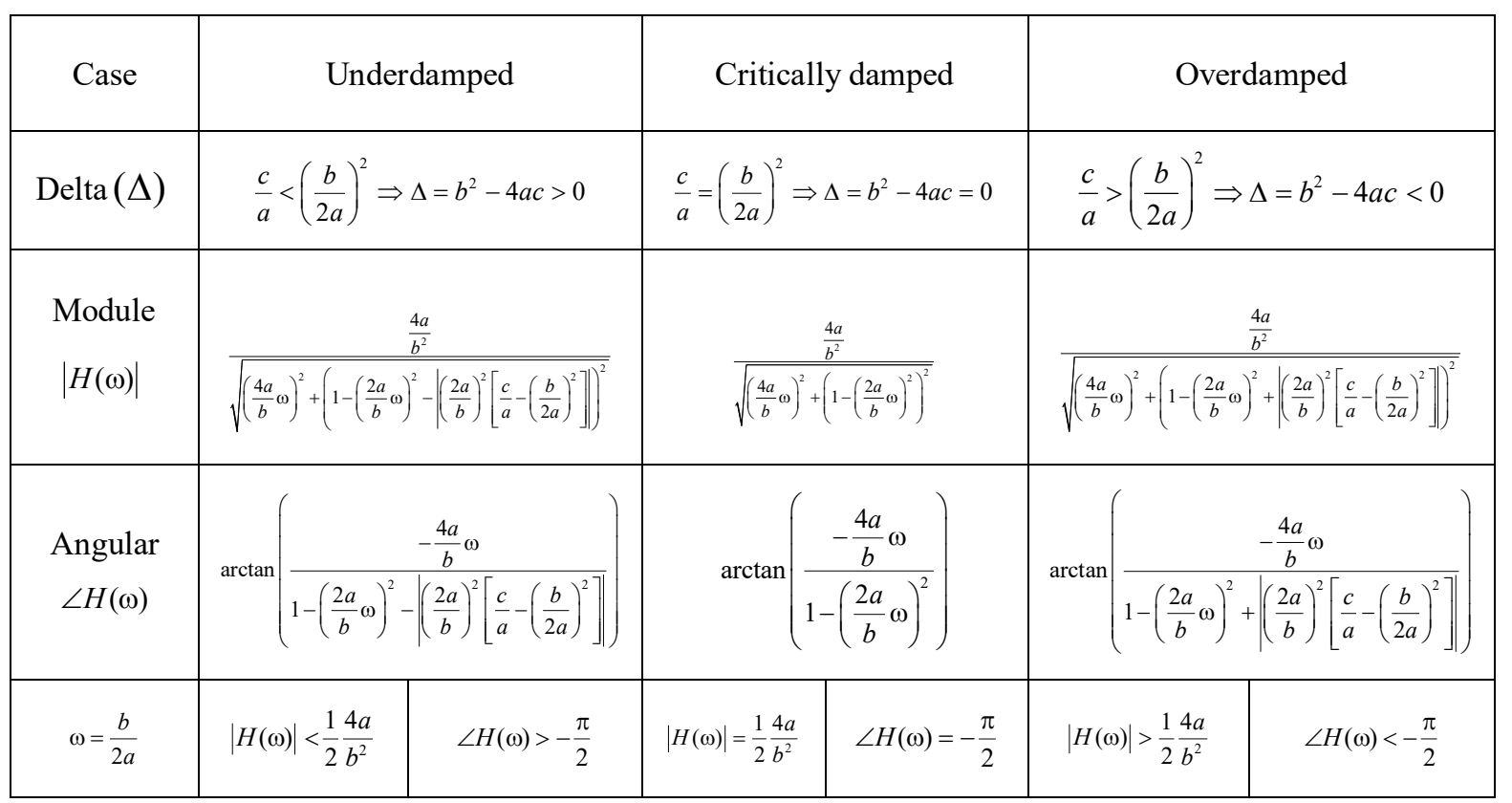




$$
\begin{aligned}
& H(j \omega)=\frac{\frac{4 a}{b^{2}}}{1+\left(\frac{2 a}{b}\right)^{2}(j \omega)^{2}+2\left(\frac{2 a}{b}\right) j \omega+\left(\frac{2 a}{b}\right)^{2}\left[\frac{c}{a}-\left(\frac{b}{2 a}\right)^{2}\right]} \\
& L(j \omega)=j \frac{4 a}{b} \omega-\left(\frac{2 a}{b} \omega\right)^{2}+\left(\frac{2 a}{b}\right)^{2}\left[\frac{c}{a}-\left(\frac{b}{2 a}\right)^{2}\right]
\end{aligned}
$$

\section{Analysis of two-stage operational amplifier}

\subsection{Transfer function for two-stage op amp}

In this section, we investigate the effects of Miller capacitor on a two-stage operational amplifier (op amp). The op amp plays an important role in an active filter ${ }^{(10)}$. In order to define the performance parameters of the second-order multiple feedbacklow-passfilter, we first take a brief look at the two-stage op amp. Fig. 6(a) shows simplified models of the two-stage op amp. As we know, frequency compensations based on Miller theory are applied in all most of two-stage op amp circuits. Let us investigate the transfer function of a two-stage op amp with frequency compensation. The gain of this topology is limited to the product of the input pair trans-conductance and the output impedance. In order to do the stability test, the transfer function at the second-stage of the two-stage op amp is considered. Furthermore, the two-stage op amp may prove inevitable if the output voltage swing must be maximized.
Thus, the stability and compensation of this op amp are of interest. Figs. 6(b) and 6(c) present the circuit and small signal model of a two-stage op amp with Miller capacitor. The transfer function and the self-loop function of this network, which are calculated in Appendix A.3., are simplified as

$$
\begin{aligned}
& H(s)=\frac{s^{3} a_{0}+s^{2} a_{1}+s a_{2}+a_{3}}{s^{4} b_{0}+s^{3} b_{1}+s^{2} b_{2}+s b_{3}+1} \\
& L(s)=s^{4} b_{0}+s^{3} b_{1}+s^{2} b_{2}+s b_{3}
\end{aligned}
$$

The values of given variables are

$$
\begin{aligned}
& a_{0}= R_{D}\left(R_{C} C_{C}\right)^{2} C_{G D} \\
& a_{1}= R_{D} R_{C} C_{C}\left(2 C_{G D}+C_{C}-g_{m} R_{C} C_{C}\right) \\
& a_{2}= R_{D}\left(C_{G D}+C_{C}-2 g_{m} R_{C} C_{C}\right) \\
& a_{3}=-g_{m} R_{D} \\
& b_{0}= R_{D} R_{S}\left(R_{C} C_{C}\right)^{2}\left[\left(C_{G D}+C_{D B}\right) C_{G S}+C_{G D} C_{D B}\right] \\
& b_{1}= R_{C} C_{C}\left\{\begin{array}{l}
R_{C} C_{C}\left[R_{D}\left(C_{G D}+C_{D B}\right)+R_{S}\left(C_{G S}+C_{G D}\right)+R_{S} R_{D} C_{G D} g_{m}\right] \\
+R_{S} R_{D}\left[2\left(C_{G S}+C_{D B}\right)\left(C_{G D}+C_{G S}\right)+C_{C} C_{G S}\right]
\end{array}\right\} \\
& b_{2}=\left\{\begin{array}{l}
R_{C} C_{C}\left[R_{C} C_{C}+2 R_{D}\left(C_{G D}+C_{D B}\right)+C_{C}\left(R_{D}+R_{S}\right)\right] \\
+R_{C}\left[2 R_{C}\left(C_{G S}+C_{G D}\right)+R_{D}\left(C_{G S}+C_{D B}\right)\right]
\end{array}\right] \\
& b_{3}=\left\{\begin{array}{l}
\left.\left(R_{C}+R_{D}\right) C_{C}+R_{D}\left(C_{G D}+C_{D B}\right)+C_{G D} C_{D B}\right]+g_{m} R_{S} R_{D} R_{C}\left(2 C_{G D}+C_{C}\right) \\
+R_{S}\left[\left(C_{G S}+C_{G D}+C_{C}\right)-g_{m} R_{D}\left(C_{G D}+C_{C}\right)\right]
\end{array}\right\}
\end{aligned}
$$

Table 2. Summary of magnitude-angular values of self-loop function

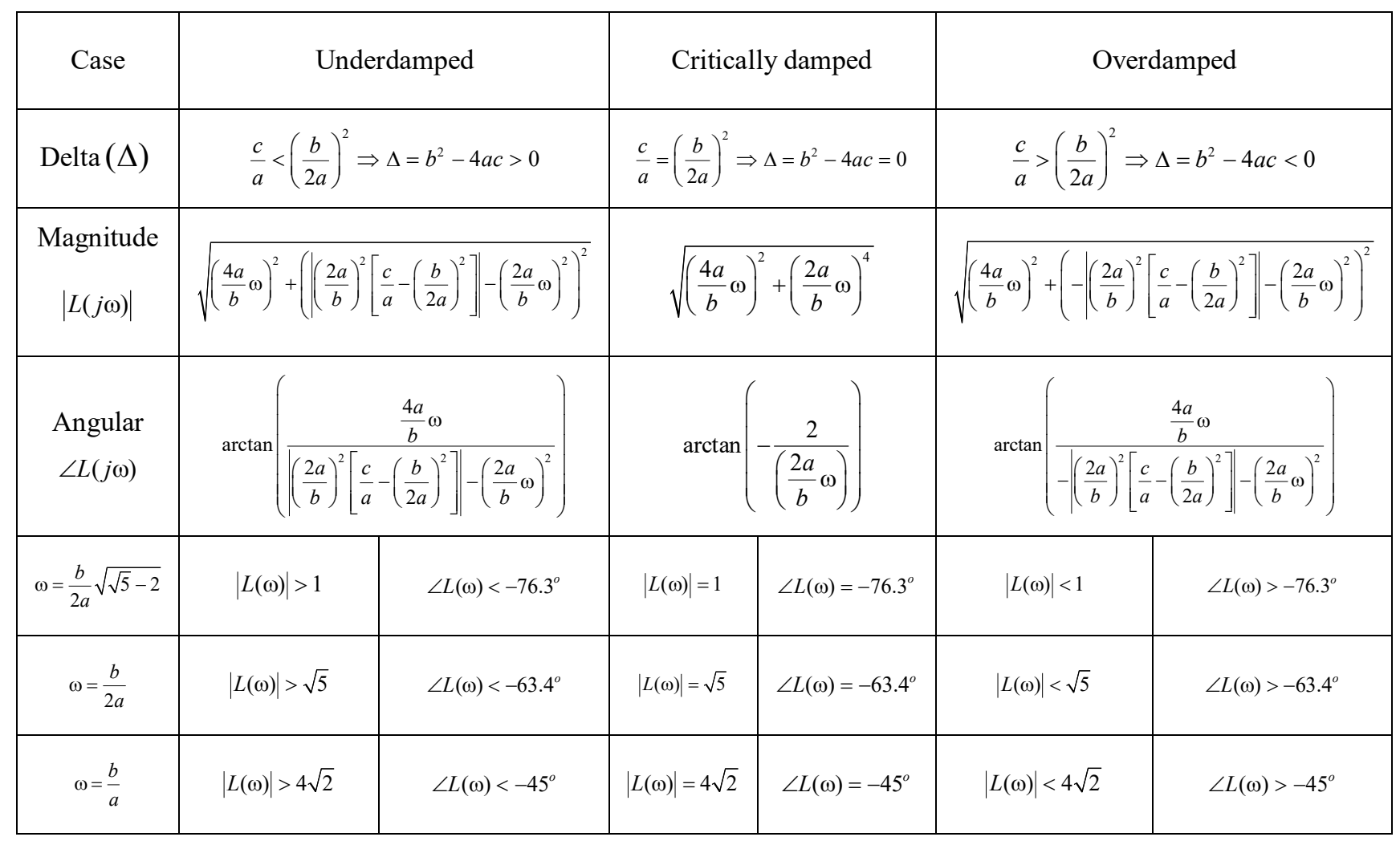



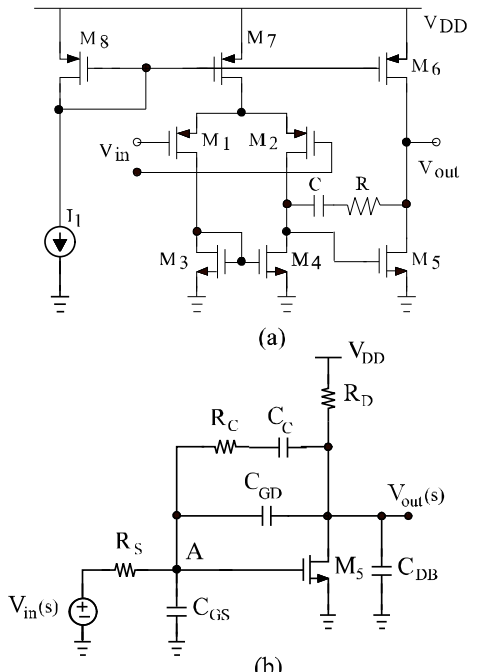

(b)

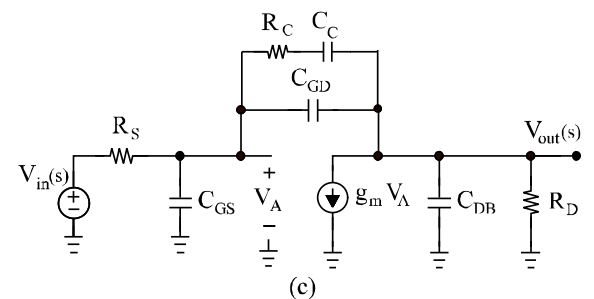

Fig. 6. Models of two-stage op amp with frequency compensation (a), second-stage of op amp (b), and small signal model (c).

When the frequency compensation is considered, the transfer function of the second-stage of the op amp is a fourth-order denominator complex function. It is very difficult to investigate the stable regions of this complex function. So, the measurement of the self-loop function is very important to do the stability test for the op amp.

\subsection{Stability test for two-stage op amp}

In this section, we do a stability test for the designed two-stage op amp.

Table 3. Device dimension.

\begin{tabular}{|c|c|c|c|c|}
\hline \multirow{4}{*}{$\begin{array}{c}\text { Transistor } \\
\text { Size }\end{array}$} & $(\mathrm{W} / \mathrm{L})_{1}$ & $(\mathrm{~W} / \mathrm{L})_{2}$ & $(\mathrm{~W} / \mathrm{L})_{3}$ & $(\mathrm{~W} / \mathrm{L})_{4}$ \\
\cline { 2 - 5 } & $18 / 0.3$ & $18 / 0.3$ & $1.6 / 0.8$ & $1.6 / 0.8$ \\
\cline { 2 - 5 } & $(\mathrm{W} / \mathrm{L})_{5}$ & $(\mathrm{~W} / \mathrm{L})_{6}$ & $(\mathrm{~W} / \mathrm{L})_{7}$ & $(\mathrm{~W} / \mathrm{L})_{8}$ \\
\cline { 2 - 5 } & $10 / 0.5$ & $1.7 / 0.3$ & $4 / 0.3$ & $1 / 0.3$ \\
\hline \multirow{2}{*}{$\begin{array}{c}\text { Capacitor } \\
\text { Value }\end{array}$} & \multicolumn{2}{|c|}{$\mathrm{C}_{1}$} & \multicolumn{2}{|c|}{$\mathrm{C}_{2}$} \\
\cline { 2 - 5 } $\begin{array}{c}\text { Resistance } \\
\text { Value }\end{array}$ & \multicolumn{2}{|c|}{$0.5 \mathrm{uF}$} & \multicolumn{2}{|c|}{$0.5 \mathrm{uF}$} \\
\cline { 2 - 5 } & \multicolumn{2}{|c|}{$\mathrm{R}_{1}$} & \multicolumn{2}{|c|}{$\mathrm{R}_{2}$} \\
\hline \multirow{2}{*}{$3 \Omega$} & \multicolumn{2}{|c|}{$10 \Omega$} \\
\hline
\end{tabular}
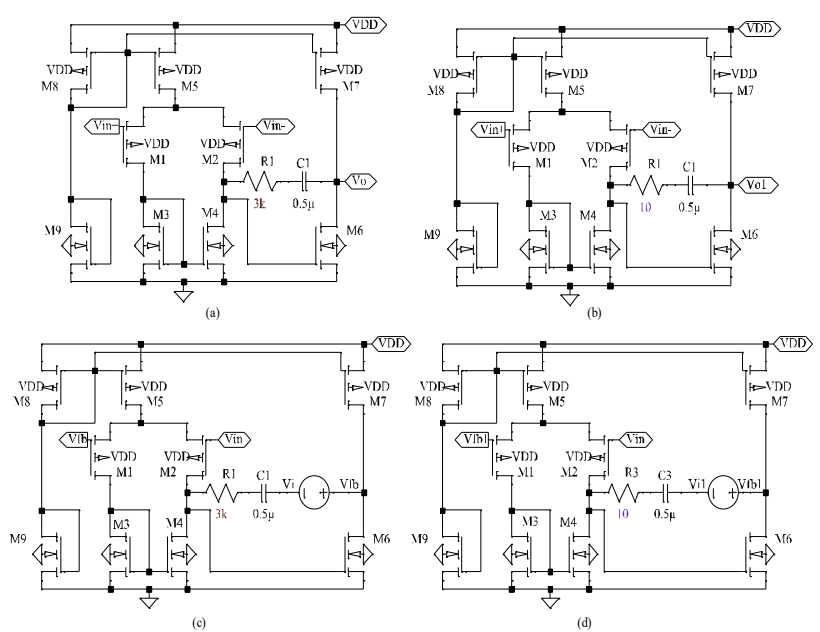

Fig. 7. Models of op amp; (a) stable, (b) unstable; derivation of self-loop function: (c) stable, (d) unstable.

The op amp circuit was simulated using SPICE Spectre simulator in TSMC 0.18um CMOS process. This op amp consumes $0.25 \mathrm{~mW}$ power from a $1.8 \mathrm{~V}$ voltage supply. All of the circuit parameters are summarized in Table 3 . Figures 7(a) and 7(b) present the models of the two-stage op amp which can be stable and unstable. The self-loop functions in these models are measured in Figures 7(c) and 7(d). In these models, the ideal capacitors and resistors are used. SPICE simulation results of the two-stage op amp are shown in Fig. 8. Based on the voltage injection technique, the self-loop functions of two-stage op amps are measured. In case of a stable op amp, the phase margin is 100 degrees at unity gain of the self-loop function.

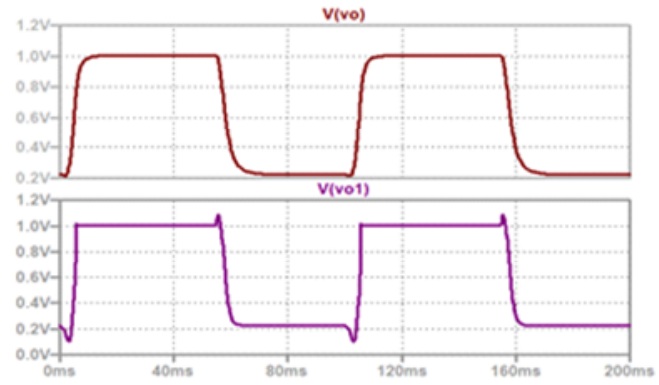

(a)

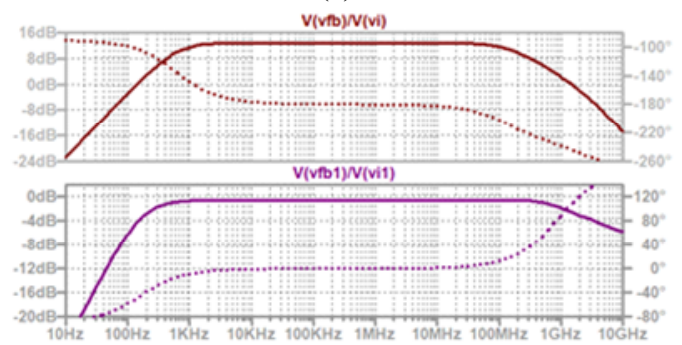

(b)

Fig. 8. Step response of op amps: (brown) stable, (purple) unstable; frequency response of self-loop functions. 
In case of an unstable op amp, the phase margin is 180 degrees at near the unity gain of the self-loop function. Therefore, the damped oscillation noise makes overshoot and undershoot.

\section{Second-order multiple feedback LPF}

\subsection{Deboo integrator}

In this section, a typical Deboo integrator is analyzed. Fig. 9 presents a model of the Deboo integrator ${ }^{(11)}$. This filter has been widely used because it is simple, versatile, and requires few components ${ }^{(12)}$. Many parameters should be considered when designing an integrator. In this topology, a single op-amp, a capacitor, and four resistors are used. The ideal operational amplifiers are used and the effect of the Miller capacitor is neglected. The transfer function of the Deboo integrator circuit, which is calculated in Appendix A.4., is derived as in Eq. (16). The simplified transfer function of this network is

$$
H(j \omega)=\frac{R_{2}\left(R_{3}+R_{4}\right)}{j \omega R_{1} R_{2} R_{3} C+R_{2} R_{3}-R_{1} R_{4}}
$$

Due to the analysis of first-order denominator complex function in Section 3, the Deboo integrator is a stable network. When $R_{2} R_{3}$ is equal to $R_{1} R_{4}$, the network is called an integrator. We have

$$
H(j \omega)=\frac{R_{3}+R_{4}}{j \omega R_{1} R_{3} C}
$$

When $R_{2} R_{3}$ is not equal to $R_{1} R_{4}$, the network is a low-pass filter. The transfer function and the self-loop function are

$$
\begin{aligned}
& H(s)=\frac{R_{2}\left(R_{3}+R_{4}\right)}{R_{2} R_{3}-R_{1} R_{4}} \frac{1}{\left(1+j \omega \frac{R_{1} R_{2} R_{3} C}{R_{2} R_{3}-R_{1} R_{4}}\right)} \\
& L(s)=j \omega \frac{R_{1} R_{2} R_{3} C}{R_{2} R_{3}-R_{1} R_{4}}
\end{aligned}
$$

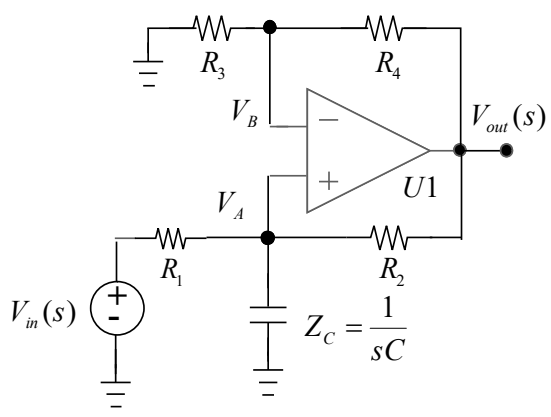

Fig. 9. Model of Deboo integrator.

\subsection{Stability test for Deboo integrator}

In this section, the proposed design and the stability test for a Deboo integrator and presented. The Deboo integrator circuit and measurement of self-loop function are shown in Fig. 10. The SPICE simulations are carried out using the ideal operational amplifier with the gain bandwidth (GBW) $=10 \mathrm{MHz}$ and $\mathrm{DC}$ value of open loop gain $(\mathrm{A}(\mathrm{s}))=100000$. The proposed model for the Deboo integrator in Fig. 10(a) is designed for unity gain at frequency $\mathrm{f}_{0}=50 \mathrm{kHz}$ taking $\mathrm{C} 1=6 \mathrm{nF}$, and $\mathrm{R} 1=\mathrm{R} 2=\mathrm{R} 3=\mathrm{R} 4=1 \mathrm{k} \Omega$. At unity gain $(4.5 \mathrm{MHz})$ of the self-loop function, the angular is 90 degrees. Compared to the simulation result with the mathematical analysis, they are the same. Therefore, the Deboo integrator is a stable network.

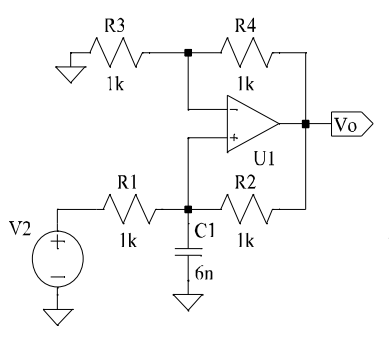

(a)

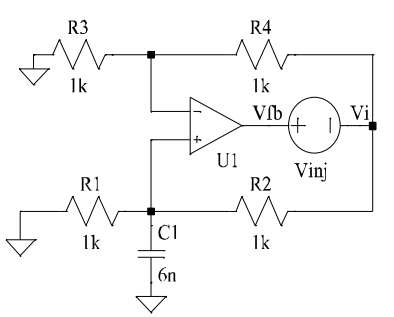

(b)
Fig. 10. Models of Deboo integrator circuit (a) and measurement of self-loop function (b).
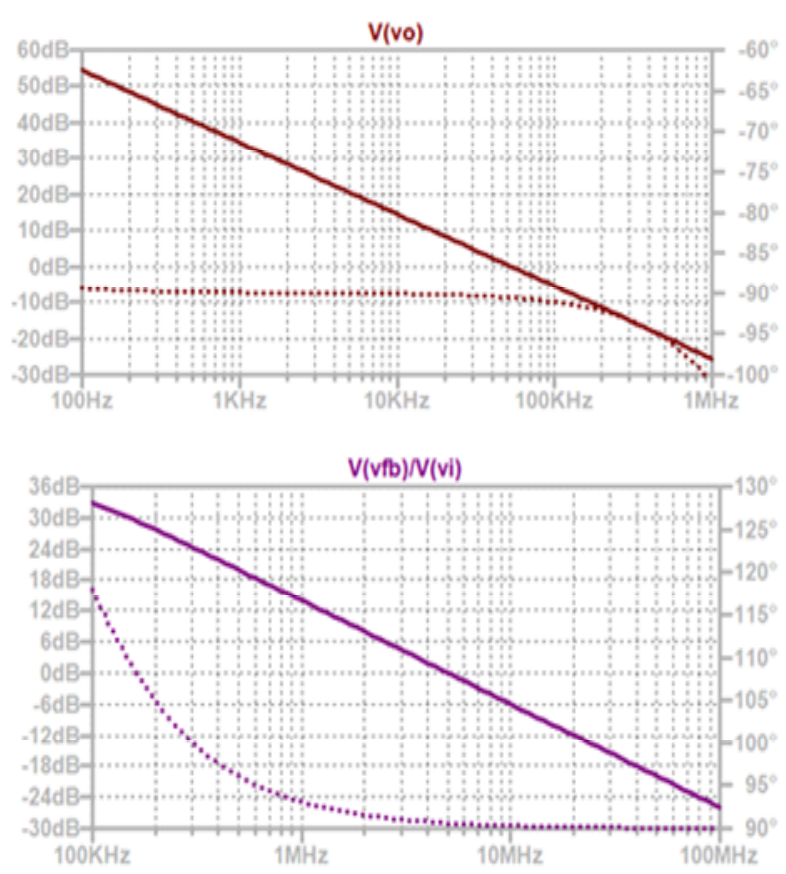

Fig. 11. Frequency response of transfer function and self-loop function. 


\subsection{Analysis of second-order multiple feedback LPF}

This section presents a second-order multiple feedback low-pass filter using the Deboo integrator. The models of the proposed design of the filter and the measurement of self-loop function are shown in Fig. 12. The transfer function and the self-loop function of this filter, which are calculated in Appendix A.5., are derived as

$$
H(s)=\frac{a_{0}}{s^{2} b_{0}+s b_{1}+1} ; L(s)=s^{2} b_{0}+s b_{1}
$$

Here, the values of given variables are

$$
\begin{aligned}
& a_{0}=-\frac{R_{2} R_{4} R_{7}\left(R_{5}+R_{6}\right)}{R_{1}\left[R_{2} R_{4}\left(R_{5}+R_{6}\right)+R_{7}\left(R_{4} R_{5}-R_{3} R_{6}\right)\right]} \\
& b_{0}=\frac{R_{2} R_{3} R_{4} R_{5} R_{7} C_{1} C_{2}}{R_{2} R_{4}\left(R_{5}+R_{6}\right)+R_{7}\left(R_{4} R_{5}-R_{3} R_{6}\right)} \\
& b_{1}=\frac{\left[R_{2} R_{7} C_{1}\left(R_{4} R_{5}-R_{3} R_{6}\right)+R_{3} R_{4} R_{5} R_{7} C_{2}\right]}{R_{2} R_{4}\left(R_{5}+R_{6}\right)+R_{7}\left(R_{4} R_{5}-R_{3} R_{6}\right)}
\end{aligned}
$$

The stability regions of the filter are defined as

$\left[\frac{R_{5}+R_{6}}{R_{3} R_{5} R_{7} C_{1} C_{2}}+\frac{R_{4} R_{5}-R_{3} R_{6}}{R_{2} R_{3} R_{4} R_{5} C_{1} C_{2}}\right]>\left[\frac{1}{2}\left(\frac{1}{R_{3} C_{2}}-\frac{R_{6}}{R_{4} R_{5} C_{2}}+\frac{1}{R_{2} C_{1}}\right)\right]^{2} \rightarrow$ Instability

$\left[\frac{R_{5}+R_{6}}{R_{3} R_{5} R_{7} C_{1} C_{2}}+\frac{R_{4} R_{5}-R_{3} R_{6}}{R_{2} R_{3} R_{4} R_{5} C_{1} C_{2}}\right]=\left[\frac{1}{2}\left(\frac{1}{R_{3} C_{2}}-\frac{R_{6}}{R_{4} R_{5} C_{2}}+\frac{1}{R_{2} C_{1}}\right)\right]^{2} \rightarrow$ Marginal stability

$$
\left[\frac{R_{5}+R_{6}}{R_{3} R_{5} R_{7} C_{1} C_{2}}+\frac{R_{4} R_{5}-R_{3} R_{6}}{R_{2} R_{3} R_{4} R_{5} C_{1} C_{2}}\right]<\left[\frac{1}{2}\left(\frac{1}{R_{3} C_{2}}-\frac{R_{6}}{R_{4} R_{5} C_{2}}+\frac{1}{R_{2} C_{1}}\right)\right]^{2} \rightarrow \text { Stability }
$$
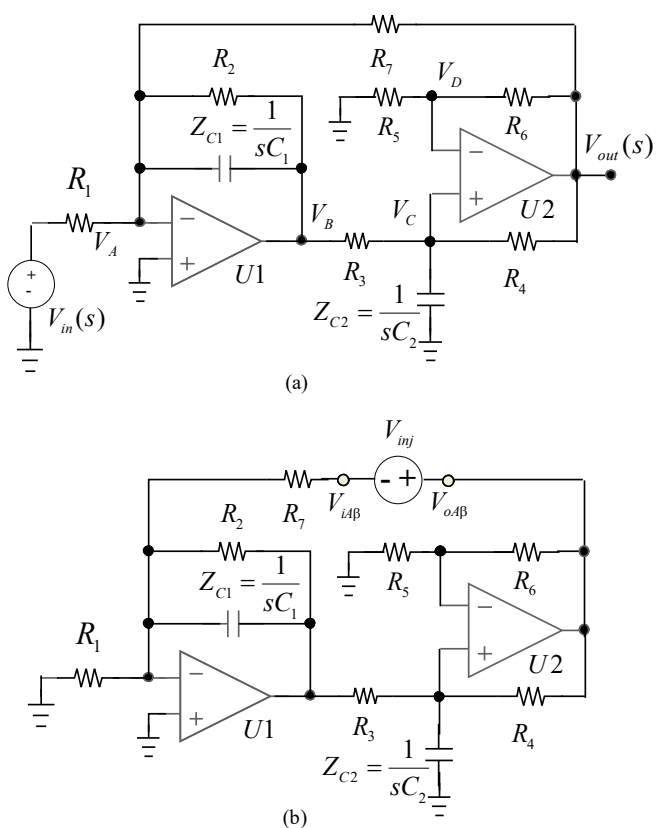

Fig. 12. Models of proposed design (a) and derivation of self-loop function (b) for multiple feedbackLPF.

\subsection{Stability test for multiple feedback LPF}

This section shows a design circuit and a stability test for a second-order multiple feedback low-pass filter using the Deboo integrator. The SPICE simulations are carried out using the ideal op amp with the gain bandwidth $(\mathrm{GBW})=$ $10 \mathrm{MHz}$ and $\mathrm{DC}$ value of open loop gain $(\mathrm{A}(\mathrm{s}))=100000$. The proposed model of the filter in Figure 13 (a) is designed for cut-off frequency $\mathrm{f}_{0}=25 \mathrm{kHz}$ taking $\mathrm{C} 1=3 \mathrm{nF}$, $\mathrm{C} 2=6 \mathrm{nF}, \mathrm{R} 1=\mathrm{R} 3=\mathrm{R} 4=\mathrm{R} 5=\mathrm{R} 6=1 \mathrm{k} \Omega, \mathrm{R} 2=1.2 \mathrm{k} \Omega$, and $\mathrm{R} 7=5 \mathrm{k} \Omega$. Figure $13(\mathrm{~b})$ represents the same circuit designed with $\mathrm{R} 2=3 \mathrm{k} \Omega$. The self-loop functions of two models are shown in Figure 13(c), (d).
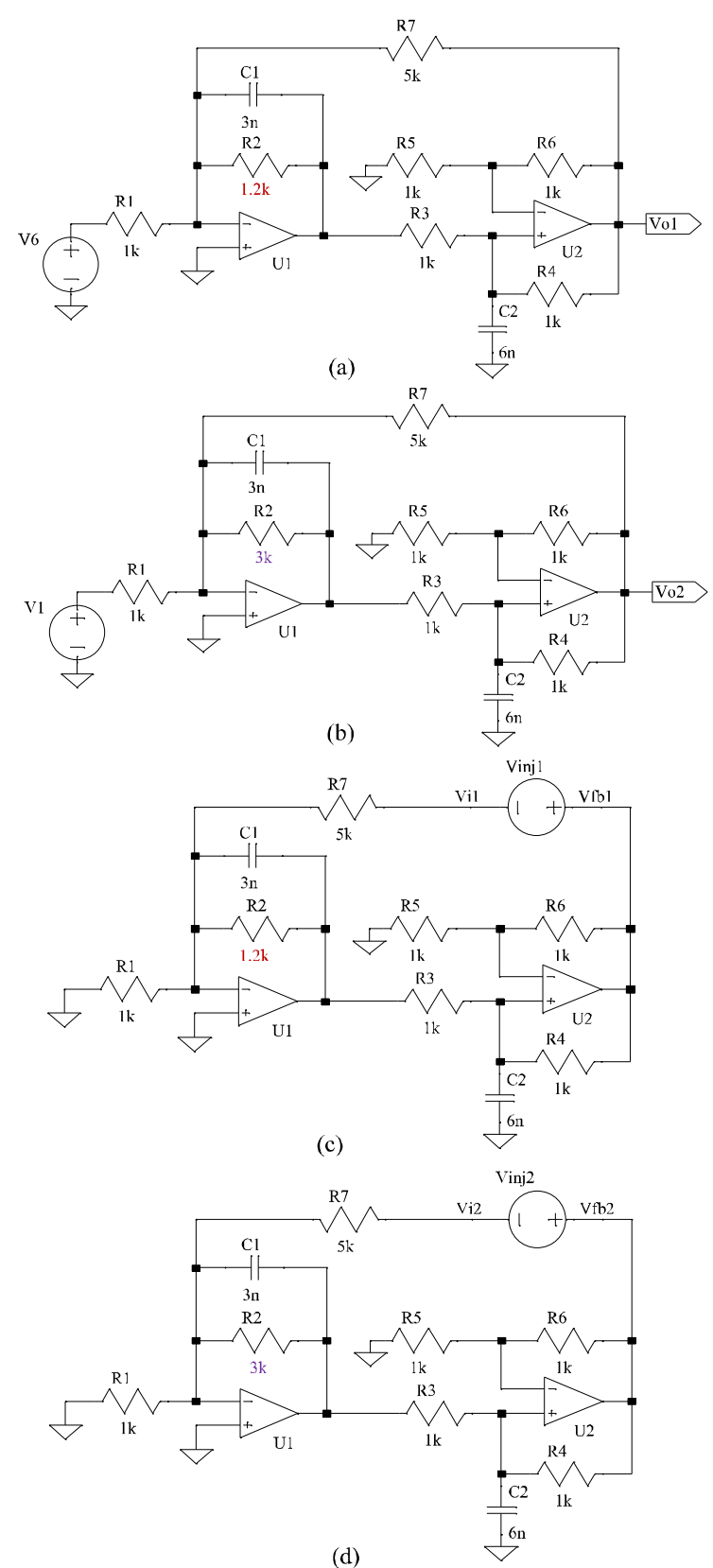

Fig. 13. Proposed models: (a) stable, and (b) unstable; derivation of self-loop function: (c) stable, (d) unstable. 


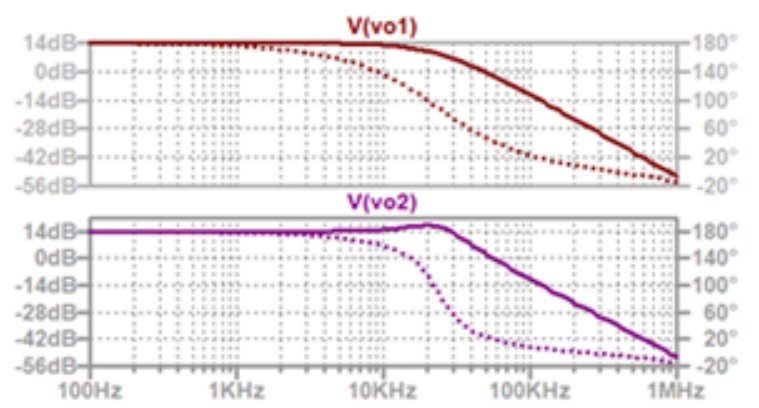

(a)

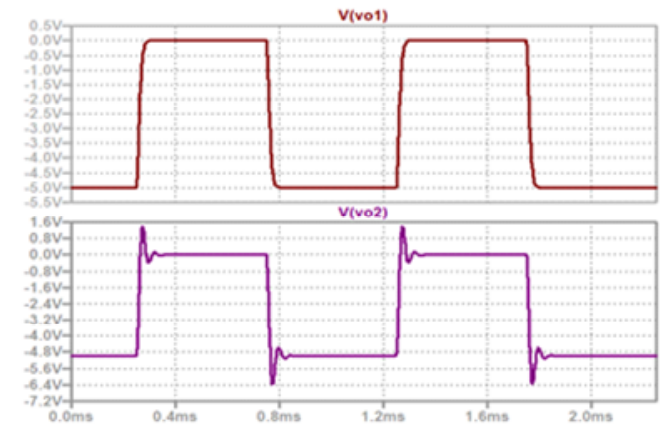

(b)

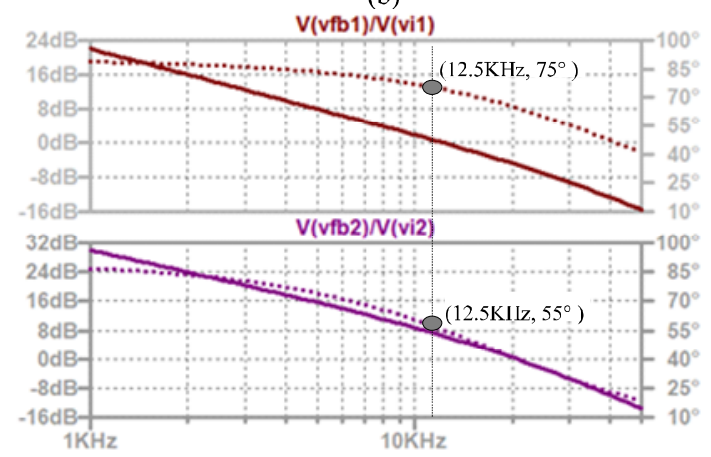

(c)

Fig. 14. Simulation results of proposeddesign: (a) frequency response, (b) transient response; (brown) stable, (purple) unstable; frequency response of self-loop function: (c) stable, and (d) unstable.

Figure 14(a) represents the SPICE simulation results of the magnitude and phase of the proposed models on the frequency domain. In time domain, when the pulse signals go in to these models, the transient responses are shown in Fig. 14(b). The damped oscillation noise (purple) occurs in case of the unstable network. The overshoot of the unstable feedback system can cause extra current to flow, thereby wasting energy and cause extra heating of the components. The measurements of the self-loop functions of the proposed models are shown in Figs. 14(c), and 14(d). In theoretical calculation at the half cut-off frequency 12.5 $\mathrm{kHz}$ (a half of $\mathrm{f}_{0}=0.5 * 25 \mathrm{kHz}=12.5 \mathrm{kHz}$ ) is 63.4 degrees. Our measurement results of self-loop functions show that
- In stable case, phase margin is 75 degrees at $12.5 \mathrm{kHz}$. ( $>63.4$ degrees at $12.5 \mathrm{kHz}$ )

- In unstable case, phase margin is 55 degrees at 12.5 $\mathrm{kHz} .(<63.4$ degrees at $12.5 \mathrm{kHz})$

The simulation results and the values of theoretical calculation are unique.

\section{Discussion}

Active filters employ op amps, resistors, and capacitors to create the desired frequency response. Second-order active sections can be based on integrators. The performance of a second-order low-pass filter, whether it has single-or multiple-loop control, is determined by its loop self-loop function and step input responses. These measurements show how good a second-order low-pass filter is. The self-loop function of a low-pass filter is only important if it gives some useful information about relative stability or if it helps optimize the transfer function performance. The self-loop function can be directly calculated based on the widened superposition principle. The alternating current conservation technique (voltage injection) can measure the self-loop function of low-pass filters. Compared to the research results with mathematical analysis, the properties of self-loop functions are the same. SPICE simulation results are included. Moreover, Nyquist theorem shows that the polar plot of self-loop function L(s) must not encircle the point $(-1,0)$ clockwise as $s$ traverses a contour around the critical region clockwise in polar chart ${ }^{(13,14)}$. However, Nyquist theorem is only used in theoretical analysis for feedback systems.

\section{Conclusions}

This paper describes the approach to do the stability test for a second-order multiple feedback low-pass filter constructed according to the Deboo's connection. The circuitry consisting of one active low-pass filter and one integrator in a feedback loop operating as a filter realizing a general biquadratic function. The transfer function of this filter is a second-order denominator complex function. Moreover, the term of "self-loop function" is proposed to define $\mathrm{L}(\mathrm{s})$ in a general transfer function. In order to show an example of how to define the operating region of a multiple feedback filter, a second-order denominator complex function is analyzed. In overdamped case, the filter will amplify the high order harmonics from the first cut-off angular frequency $\omega_{\text {cut } 1}$ to the second cut-off angular 
frequency $\omega_{\text {cut2 }}$ of a step input. This causes the unwanted noise which is called ringing or overshoot. The term of "damped oscillation noise" is proposed to define the ringing. In this work, the values of the passive components used in the second-order multiple feedback low-passfilter circuit were chosen directly due to the stable conditions. All of the transfer functions were derived based on the widened superposition principle and self-loop functions were measured according to the alternating current conservation technique. The obtained results were acquired to simulations using SPICE models of the devices, including the model of a two-stage operational amplifier. In this paper not only the results of the mathematical model but also the results of simulation of the designed circuits are provided, including the stability test. The simulation results and the values of theoretical calculation of the self-loop function are unique. Furthermore, managing power consumption of circuits and systems is one of the most important challenges for the semiconductor industry ${ }^{(15)}$. Therefore, the damped oscillation noise test can be used to evaluate the stability of a feedback system.

\section{Acknowledgment}

Foremost, we would like to express our sincere gratitude to advisor, Pro. Tanimoto (Kitami Institute. of Technology, Japan) for continuing supports.

\section{References}

(1) H. Kobayashi, M. Tran, K.Asami, A.Kuwana, H. San, "Complex Signal Processing in Analog, Mixed - Signal Circuits", Proceedings of International Conference on Technology and Social Science 2019, (Kiryu, Japan) May. 2019.

(2) R. Schaumann, M. Valkenberg, Design of Analog Filters, Oxford University Press, 2001.

(3) C. Huynh, C. Nguyen, "Design of CMOS RFIC Transmitters for Wireless Communications and Sensing", John-Wiley \& Sons, New York, to be published 2013.

(4) M. Tran, C. Huynh, "A Design of RF Front-End for ZigBee Receiver using Low-IF architecture with Poly-phase Filter for Image Rejection", M.S. thesis, University of Technology Ho Chi Minh City - Vietnam, Dec. 2014.

(5) S. Rosenstark, Feedback Amplifiers Principles,MacMillan, New York, 1986.
(6) M. Tran, N. Kushita, A. Kuwana, H. Kobayashi,"Flat Pass-Band Method with Two RC Band-Stop Filters for 4-Stage Passive RC Quadratic Filter in Low-IF Receiver Systems", IEEE 13th International Conference on ASIC (ASICON 2019) (Chongqing, China), Nov, 2019.

(7) M. Tran, Y. Sun, N. Oiwa, Y. Kobori, A. Kuwana, H. Kobayashi, "Mathematical Analysis and Design of Parallel RLC Network in Step-down Switching Power Conversion System", Proceedings of International Conference on Technology and Social Science ICTSS 2019 (Kiryu, Japan) (May. 2019).

(8) B. Razavi, Design of Analog CMOS Integrated Circuits, 2nd Edition McGraw-Hill (2016).

(9) M. Tran, Y. Sun, Y. Kobori, A. Kuwana, H. Kobayashi, "Overshoot Cancelation Based on Balanced Charge-Discharge Time Condition for Buck Converter in Mobile Applications", IEEE $13^{\text {th }}$ International Conference on ASIC (ASICON 2019) (Chongqing, China), Nov, 2019.

(10) R. Middlebrook, "Measurement of Loop Gain in Feedback Systems", Int. J. Electronics, vol 38, No. 4, pp. 485-512, 1975.

(11) G. Deboo, "A Novel Integrator Results by Grounding its Capacitor", Electronic Design, vol. 15, June 1967.

(12) A. Sedra, K. Smith, Microelectronic Circuits, 6th ed. Oxford University Press, New York, 2010.

(13) M. Tran, "Damped Oscillation Noise Test for Feedback Circuit Based on Comparison Measurement Technique", $73^{\text {rd }}$ System LSI Joint Seminar, Tokyo Institute of Technology, (Tokyo, Japan), Oct, 2019.

(14) P. Allen, D. Holberg, CMOS Analog Circuit Design, $2^{\text {nd }}$ ed., Oxford University Press, New York, 2002.

(15)H. Kobayashi, N. Kushita, M. Tran, K. Asami, H. San, A. Kuwana, "Analog - Mixed-Signal - RF Circuits for Complex Signal Processing", IEEE 13th International Conference on ASIC (ASICON 2019) (Chongqing, China), Nov, 2019.

\section{Appendix}

\section{A.1. Second-order denominator complex function}

From Eq. (11), the transfer function is rewritten as

$$
H(s)=\frac{1}{a s^{2}+b s+c}=\frac{\frac{1}{a}}{s^{2}+2\left(\frac{b}{2 a}\right) s+\left(\frac{b}{2 a}\right)^{2}+\left[\frac{c}{a}-\left(\frac{b}{2 a}\right)^{2}\right]}
$$


The simplified form of Eq. (25) is

$$
\begin{aligned}
H(s=j \omega) & =\frac{\frac{4 a}{b^{2}}}{\left(1+\frac{2 a}{b} s\right)^{2}+\left(\frac{2 a}{b}\right)^{2}\left[\frac{c}{a}-\left(\frac{b}{2 a}\right)^{2}\right]} \\
& =\frac{\frac{4 a}{b^{2}}}{\left(1+j \frac{2 a}{b} \omega\right)^{2}+\left(\frac{2 a}{b}\right)^{2}\left[\frac{c}{a}-\left(\frac{b}{2 a}\right)^{2}\right]}
\end{aligned}
$$

The magnitude-angular form of transfer function is

$$
\begin{aligned}
& |H(j \omega)|=\frac{4 a}{b^{2}} \frac{1}{\sqrt{\left(\frac{4 a}{b} \omega\right)^{2}+\left(1-\left(\frac{2 a}{b} \omega\right)^{2}+\left(\frac{2 a}{b}\right)^{2}\left[\frac{c}{a}-\left(\frac{b}{2 a}\right)^{2}\right]\right)^{2}}} \\
& \angle H(j \omega)=\arctan \left(\frac{-\frac{4 a}{b} \omega}{1-\left(\frac{2 a}{b} \omega\right)^{2}+\left(\frac{2 a}{b}\right)^{2}\left[\frac{c}{a}-\left(\frac{b}{2 a}\right)^{2}\right]}\right)
\end{aligned}
$$

In critically damped case $\frac{c}{a}=\left(\frac{b}{2 a}\right)^{2}$, the magnitude-angular form of transfer function is

$$
|H(j \omega)|=\frac{4 a}{b^{2}} \frac{1}{\sqrt{\left(\frac{4 a}{b} \omega\right)^{2}+\left(1-\left(\frac{2 a}{b} \omega\right)^{2}\right)^{2}}} ; \angle H(j \omega)=\arctan \left(\frac{-\frac{4 a}{b} \omega}{1-\left(\frac{2 a}{b} \omega\right)^{2}}\right)
$$

At the cut-off angular frequency $\omega_{c u t}=\frac{b}{2 a}$, the magnitude-angular form of transfer function is

$$
|H(j \omega)|=\frac{2 a}{b^{2}} ; \angle H(j \omega)=\arctan (-\infty)=-\frac{\pi}{2}
$$

\section{A.2. Self-loop function of second-order denominator complex function}

From Eq. (14), the self-loop function is rewritten as

$$
L(j \omega)=j \frac{4 a}{b} \omega+\left(j \frac{2 a}{b} \omega\right)^{2}+\left(\frac{2 a}{b}\right)^{2}\left[\frac{c}{a}-\left(\frac{b}{2 a}\right)^{2}\right]
$$

The magnitude-angular form of self-loop function is

$$
\begin{aligned}
& |L(j \omega)|=\sqrt{\left(\frac{4 a}{b} \omega\right)^{2}+\left(\left(\frac{2 a}{b}\right)^{2}\left[\frac{c}{a}-\left(\frac{b}{2 a}\right)^{2}\right]-\left(\frac{2 a}{b} \omega\right)^{2}\right)^{2}} \\
& \angle L(j \omega)=\arctan \left(\frac{\frac{4 a}{b} \omega}{\left(\frac{2 a}{b}\right)^{2}\left[\frac{c}{a}-\left(\frac{b}{2 a}\right)^{2}\right]-\left(\frac{2 a}{b} \omega\right)^{2}}\right)
\end{aligned}
$$

In critically damped case $\frac{c}{a}=\left(\frac{b}{2 a}\right)^{2}$, the self-loop function is

$$
L(j \omega)=j \frac{4 a}{b} \omega\left(1+j \frac{a}{b} \omega\right)={\sqrt{\left(\frac{4 a}{b} \omega\right)^{2}+\left(\frac{2 a}{b} \omega\right)^{4}}}^{j \arctan \left(\frac{2}{-\left(\frac{2 a}{b} \omega\right)}\right)}
$$

At the angular frequency $\omega=\frac{b}{a}$, the magnitude-angular form of self-loop function is

$$
|L(j \omega)|=4 \sqrt{2} ; \quad L L(\omega)=\arctan (-1)=-45^{\circ}
$$

At the angular frequency $\omega_{c u t}=\frac{b}{2 a}$, the magnitude-angular form of self-loop function is

$$
|L(j \omega)|=\sqrt{5} ; \angle L(j \omega)=\arctan (-2)=-63.4^{\circ}
$$

At unity gain of the self-loop function, we have

$$
\left|L\left(\omega_{u}\right)\right|=1 \Rightarrow\left|\frac{4 a}{b} \omega_{u} \sqrt{1+\left(\frac{a}{b} \omega_{u}\right)^{2}}\right|=1
$$

Solving Eq. (33), the angular frequency $\omega_{u}$ at unity gain is calculated as

$$
\omega_{u}=\frac{b}{2 a} \sqrt{\sqrt{5}-2}
$$

The relationship between the angular frequency $\omega_{u}$ and the cut-off angular frequency $\omega_{c u t}=\frac{b}{2 a}$ is

$$
\omega_{u}=\omega_{c u t} \sqrt{\sqrt{5}-2} \Rightarrow \omega_{c u t}=\frac{\omega_{u}}{\sqrt{\sqrt{5}-2}}
$$

At unity gain angular frequency $\omega_{u}=\frac{b}{2 a} \sqrt{\sqrt{5}-2}$, the magnitude-angular form of self-loop function is

$$
|L(j \omega)|=1 ; \angle L(j \omega)=\arctan \left(\frac{-2}{\sqrt{\sqrt{5}-2}}\right)=-76.35^{\circ}
$$

In underdamped case $\frac{c}{a}<\left(\frac{b}{2 a}\right)^{2}$, the self-loop function is 


$$
L(j \omega)=j \frac{4 a}{b} \omega-\left(j \frac{2 a}{b} \omega\right)^{2}-\left|\left(\frac{2 a}{b}\right)^{2}\left[\frac{c}{a}-\left(\frac{b}{2 a}\right)^{2}\right]\right|
$$

The magnitude-angular form of self-loop function is

$$
\begin{aligned}
& |L(j \omega)|=\sqrt{\left(\frac{4 a}{b} \omega\right)^{2}+\left(-\left|\left(\frac{2 a}{b}\right)^{2}\left[\frac{c}{a}-\left(\frac{b}{2 a}\right)^{2}\right]\right|-\left(\frac{2 a}{b} \omega\right)^{2}\right)^{2}} \\
& \angle L(j \omega)=\arctan \left(\frac{\frac{4 a}{b} \omega}{-\left(\frac{2 a}{b}\right)^{2}\left[\frac{c}{a}-\left(\frac{b}{2 a}\right)^{2}\right] \mid-\left(\frac{2 a}{b} \omega\right)^{2}}\right)
\end{aligned}
$$

At the angular frequency $\omega=\frac{b}{a}$, the magnitude-angular form of self-loop function is

$$
\begin{aligned}
& |L(j \omega)|=\sqrt{(4)^{2}+\left(-\left|\left(\frac{2 a}{b}\right)^{2}\left[\frac{c}{a}-\left(\frac{b}{2 a}\right)^{2}\right]\right|-(4)^{2}\right)^{2}}>4 \sqrt{2} \\
& \angle L(j \omega)=\arctan \left(\frac{4}{\left.-\left|\left(\frac{2 a}{b}\right)^{2}\left[\frac{c}{a}-\left(\frac{b}{2 a}\right)^{2}\right]\right|-4\right)}<\arctan (-1)=-45^{\circ}\right.
\end{aligned}
$$

At the angular frequency $\omega_{c u t}=\frac{b}{2 a}$, the magnitude-angular form of self-loop function is

$$
\begin{aligned}
& |L(j \omega)|=\sqrt{4+\left(-\left|\left(\frac{2 a}{b}\right)^{2}\left[\frac{c}{a}-\left(\frac{b}{2 a}\right)^{2}\right]\right|-1\right)^{2}}>\sqrt{5} \\
& \angle L(j \omega)=\arctan \left(\frac{2}{-\left|\left(\frac{2 a}{b}\right)^{2}\left[\frac{c}{a}-\left(\frac{b}{2 a}\right)^{2}\right]\right|-1}\right)<\arctan (-2)=-63.4^{\circ}
\end{aligned}
$$

At unity gain angular frequency $\omega_{u}=\frac{b}{2 a} \sqrt{\sqrt{5}-2}$, the magnitude-angular form of self-loop function is

$$
\begin{aligned}
& |L(j \omega)|=\sqrt{2(\sqrt{5}-2)+\left(-\left(\frac{2 a}{b}\right)^{2}\left[\frac{c}{a}-\left(\frac{b}{2 a}\right)^{2}\right] \mid+2-\sqrt{5}\right)^{2}}>1 \\
& \operatorname{Arg}\{L(j \omega)\}=\frac{2 \sqrt{\sqrt{5}-2}}{-\mid\left(\frac{2 a}{b}\right)^{2}\left[\frac{c}{a}-\left(\frac{b}{2 a}\right)^{2}\right]-(\sqrt{5}-2)}<\arctan \left(\frac{-2}{\sqrt{\sqrt{5}-2}}\right)=-76.35^{\circ}
\end{aligned}
$$

In overdamped case $\frac{c}{a}>\left(\frac{b}{2 a}\right)^{2}$, the self-loop function is

$$
L(j \omega)=j \frac{4 a}{b} \omega-\left(j \frac{2 a}{b} \omega\right)^{2}+\left|\left(\frac{2 a}{b}\right)^{2}\left[\frac{c}{a}-\left(\frac{b}{2 a}\right)^{2}\right]\right|
$$

The magnitude-angular form of self-loop function is

$$
\begin{aligned}
& |L(j \omega)|=\sqrt{\left(\frac{4 a}{b} \omega\right)^{2}+\left(\left|\left(\frac{2 a}{b}\right)^{2}\left[\frac{c}{a}-\left(\frac{b}{2 a}\right)^{2}\right]\right|-\left(\frac{2 a}{b} \omega\right)^{2}\right)^{2}} \\
& \angle L(j \omega)=\arctan \left(\frac{\frac{4 a}{b} \omega}{\mid\left(\frac{2 a}{b}\right)^{2}\left[\frac{c}{a}-\left(\frac{b}{2 a}\right)^{2}\right]-\left(\frac{2 a}{b} \omega\right)^{2}}\right)
\end{aligned}
$$

At the angular frequency $\omega=\frac{b}{a}$, the magnitude-angular form of self-loop function is

$$
\begin{aligned}
& |L(j \omega)|=\sqrt{(4)^{2}+\left(\left|\left(\frac{2 a}{b}\right)^{2}\left[\frac{c}{a}-\left(\frac{b}{2 a}\right)^{2}\right]\right|-(4)^{2}\right)^{2}}<4 \sqrt{2} \\
& \angle L(j \omega)=\arctan \left(\frac{4}{\left|\left(\frac{2 a}{b}\right)^{2}\left[\frac{c}{a}-\left(\frac{b}{2 a}\right)^{2}\right]\right|-4}\right)>\arctan (-1)=-45^{\circ}
\end{aligned}
$$

At the angular frequency $\omega_{c u t}=\frac{b}{2 a}$, the magnitude-angular form of self-loop function is

$$
\begin{aligned}
& |L(j \omega)|=\sqrt{4+\left(\left|\left(\frac{2 a}{b}\right)^{2}\left[\frac{c}{a}-\left(\frac{b}{2 a}\right)^{2}\right]\right|-1\right)^{2}}<\sqrt{5} \\
& \angle L(j \omega)=\arctan \left(\frac{2}{\left|\left(\frac{2 a}{b}\right)^{2}\left[\frac{c}{a}-\left(\frac{b}{2 a}\right)^{2}\right]\right|-1}\right)>\arctan (-2)=-63.4^{\circ}
\end{aligned}
$$

At unity gain angular frequency $\omega_{u}=\frac{b}{2 a} \sqrt{\sqrt{5}-2}$, the magnitude-angular form of self-loop function is

$$
\begin{aligned}
& |L(j \omega)|=\sqrt{2(\sqrt{5}-2)+\left(\left|\left(\frac{2 a}{b}\right)^{2}\left[\frac{c}{a}-\left(\frac{b}{2 a}\right)^{2}\right]\right|+2-\sqrt{5}\right)^{2}}<1 \\
& \angle L(j \omega)=\arctan \left(\frac{2 \sqrt{\sqrt{5}-2}}{\left|\left(\frac{2 a}{b}\right)^{2}\left[\frac{c}{a}-\left(\frac{b}{2 a}\right)^{2}\right]\right|-(\sqrt{5}-2)}\right)>\arctan \left(\frac{-2}{\sqrt{\sqrt{5}-2}}\right)=-76.35^{\circ}
\end{aligned}
$$

\section{A.3. Small signal model of second stage of op amp}

Apply the widened superposition at $\mathrm{V}_{\mathrm{A}}$ node, we get

$$
V_{A}\left(\frac{1}{R_{S}}+\frac{1}{Z_{C G S}}+\frac{1}{Z_{C G D}}+\frac{1}{R_{C}+Z_{C C}}\right)=\frac{V_{\text {in }}}{R_{S}}+V_{\text {out }}\left(\frac{1}{Z_{C G D}}+\frac{1}{R_{C}+Z_{C C}}\right)
$$




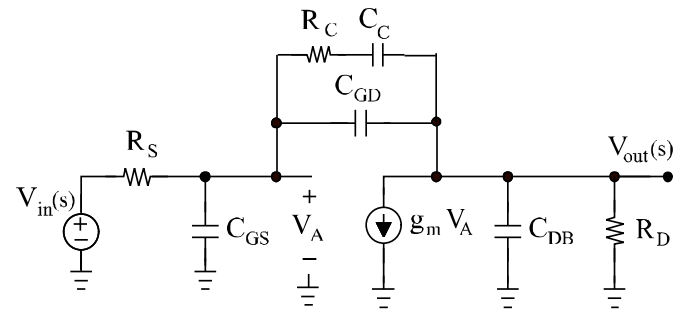

Fig. 14. Circuit of Figure 6(c).

Then, apply the widened superposition at $\mathrm{V}_{\text {out }}$ node, we get $V_{\text {out }}\left(\frac{1}{Z_{C G D}}+\frac{1}{R_{C}+Z_{C C}}+\frac{1}{Z_{C D B}}+\frac{1}{R_{D}}\right)=V_{A}\left(\frac{1}{Z_{C G D}}+\frac{1}{R_{C}+Z_{C C}}-g_{m}\right)$

The transfer function of this network is

$$
\begin{aligned}
& H(s)=\frac{\left(\frac{1}{Z_{C D D}}+\frac{1}{R_{C}+Z_{C C}}-g_{m}\right)}{\left(\frac{1}{Z_{C D D}}+\frac{1}{R_{C}+Z_{C C}}+\frac{1}{Z_{C D B}}+\frac{1}{R_{D}}\right)+R_{s}\left[\begin{array}{l}
\left(\frac{1}{Z_{C D D}}+\frac{1}{R_{C}+Z_{C C}}+\frac{1}{Z_{C D B}}+\frac{1}{R_{D}}\right)\left(\frac{1}{Z_{C D S}}+\frac{1}{Z_{C C D}}+\frac{1}{R_{C}+Z_{C C}}\right) \\
-\left(\frac{1}{Z_{C D D}}+\frac{1}{R_{C}+Z_{C C}}-g_{m}\right)\left(\frac{1}{Z_{C D D}}+\frac{1}{R_{C}+Z_{C C}}\right)
\end{array}\right]} \\
& =\frac{\left(s C_{C D}+\frac{s C_{C}}{s R_{C} C_{C}+1}-g_{m}\right)}{\left(s C_{C D}+\frac{s C_{C}}{s R_{C} C_{C}+1}+s C_{D B}+\frac{1}{R_{D}}\right)+R_{S}\left(\begin{array}{l}
\left.\left(s C_{C D}+\frac{s C_{C}}{s R_{C} C_{C}+1}+s C_{D B}+\frac{1}{R_{D}}\right)\left(s C_{C S}+s C_{C D}+\frac{s C_{C}}{s R_{C} C_{C}+1}\right)\right] \\
-\left(s C_{C D}+\frac{s C_{C}}{s R_{C} C_{C}+1}-g_{m}\right)\left(s C_{G D}+\frac{s C_{C}}{s R_{C} C_{C}+1}\right)
\end{array}\right]}
\end{aligned}
$$

Now, the simplified transfer function of this network is

$$
H(s)=\frac{\left[\begin{array}{l}
s^{3} R_{D}\left(R_{C} C_{C}\right)^{2} C_{G D}+s^{2} R_{D} R_{C} C_{C}\left(2 C_{G D}+C_{C}-g_{m} R_{C} C_{C}\right) \\
+s R_{D}\left(C_{G D}+C_{C}-2 g_{m} R_{C} C_{C}\right)-g_{m} R_{D}
\end{array}\right]}{\left\{\begin{array}{l}
s^{4} R_{D} R_{S}\left(R_{C} C_{C}\right)^{2}\left[\left(C_{G D}+C_{D B}\right) C_{G S}+C_{G D} C_{D B}\right] \\
+s^{3} R_{C} C_{C}\left\{\begin{array}{l}
R_{C} C_{C}\left[R_{D}\left(C_{G D}+C_{D B}\right)+R_{S}\left(C_{G S}+C_{G D}\right)+R_{S} R_{D} C_{G D} g_{m}\right] \\
+R_{S} R_{D}\left[2\left(C_{G S}+C_{D B}\right)\left(C_{G D}+C_{G S}\right)+C_{C} C_{G S}\right]
\end{array}\right\} \\
+s^{2}\left\{\begin{array}{l}
R_{C} C_{C}\left[R_{C} C_{C}+2 R_{D}\left(C_{G D}+C_{D B}\right)+C_{C}\left(R_{D}+R_{S}\right)\right] \\
+R_{S} C_{C}\left[2 R_{C}\left(C_{G S}+C_{G D}\right)+R_{D}\left(C_{G S}+C_{D B}\right)\right] \\
+R_{S} R_{D}\left[C_{G S}\left(C_{G D}+C_{D B}\right)+C_{G D} C_{D B}\right]+g_{m} R_{S} R_{D} R_{C}\left(2 C_{G D}+C_{C}\right)
\end{array}\right\} \\
+s\left\{\begin{array}{l}
\left(R_{C}+R_{D}\right) C_{C}+R_{D}\left(C_{G D}+C_{D B}\right)+R_{C} C_{C} \\
+R_{S}\left[\left(C_{G S}+C_{G D}+C_{C}\right)-g_{m} R_{D}\left(C_{G D}+C_{C}\right)\right]
\end{array}\right\}+1
\end{array}\right\}}
$$

\section{A.4. Deboo integrator}

Apply the widened superposition at $\mathrm{V}_{\mathrm{A}}$ node, we get

$$
V_{A}\left(\frac{1}{R_{1}}+\frac{1}{Z_{C}}+\frac{1}{R_{2}}\right)=\frac{V_{\text {in }}}{R_{1}}+\frac{V_{\text {out }}}{R_{2}}
$$

Do the same work at $\mathrm{V}_{\mathrm{B}}$ node, we get

$$
V_{B}\left(\frac{1}{R_{3}}+\frac{1}{R_{4}}\right)=\frac{V_{\text {out }}}{R_{4}}
$$

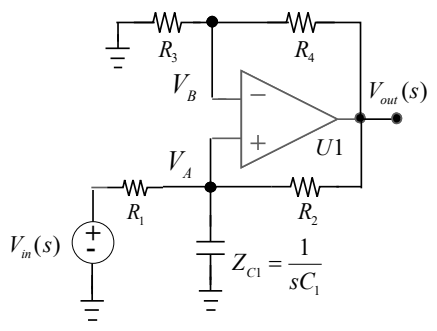

Fig. 15. Circuit of Figure 9.

Here, $V_{A}=V_{B}$, the simplified transfer function of this network is

$$
H(s)=\frac{R_{2} Z_{C}\left(R_{3}+R_{4}\right)}{Z_{C}\left(R_{2} R_{3}-R_{1} R_{4}\right)+R_{1} R_{2} R_{3}}=\frac{R_{2}\left(R_{3}+R_{4}\right)}{s R_{1} R_{2} R_{3} C+R_{2} R_{3}-R_{1} R_{4}}
$$

\section{A.5. Second-order multiple feedback LPF}

Apply the widened superposition at node $V_{A}$, we get

$V_{A}\left(\frac{1}{R_{1}}+\frac{1}{Z_{C 1}}+\frac{1}{R_{2}}+\frac{1}{R_{7}}\right)=\frac{V_{\text {in }}}{R_{1}}+V_{B}\left(\frac{1}{R_{2}}+\frac{1}{Z_{C 1}}\right)+\frac{V_{\text {out }}}{R_{7}}=0$

Do the same work at node $V_{C}$, we get

$$
V_{C}\left(\frac{1}{R_{3}}+\frac{1}{Z_{C 2}}+\frac{1}{R_{4}}\right)=\frac{V_{B}}{R_{3}}+\frac{V_{\text {out }}}{R_{4}}
$$

Then at node $\mathrm{V}_{\mathrm{D}}$, we get

$$
V_{D}\left(\frac{1}{R_{5}}+\frac{1}{R_{6}}\right)=\frac{V_{\text {out }}}{R_{6}}
$$

The simplified transfer function of this filter is derived as

$$
H(s)=\frac{-\frac{R_{2} R_{4} R_{7}\left(R_{5}+R_{6}\right)}{R_{1}}}{\left\{\begin{array}{l}
s^{2} R_{2} R_{3} R_{4} R_{5} R_{7} C_{1} C_{2}+s\left[\begin{array}{l}
R_{2} R_{7} C_{1}\left(R_{4} R_{5}-R_{3} R_{6}\right) \\
+R_{3} R_{4} R_{5} R_{7} C_{2}
\end{array}\right] \\
+R_{2} R_{4}\left(R_{5}+R_{6}\right)+R_{7}\left(R_{4} R_{5}-R_{3} R_{6}\right)
\end{array}\right\}}
$$

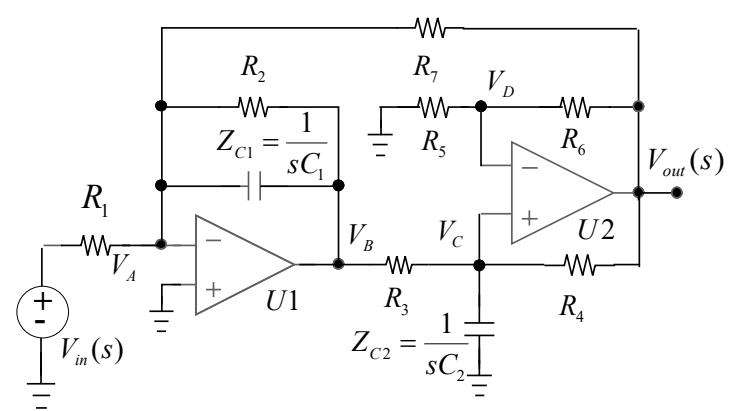

Fig. 16. Circuit of Figure 12(a). 